\title{
Multidisciplinary laparo-endoscopic management of a subcardial gastric diverticulum
}

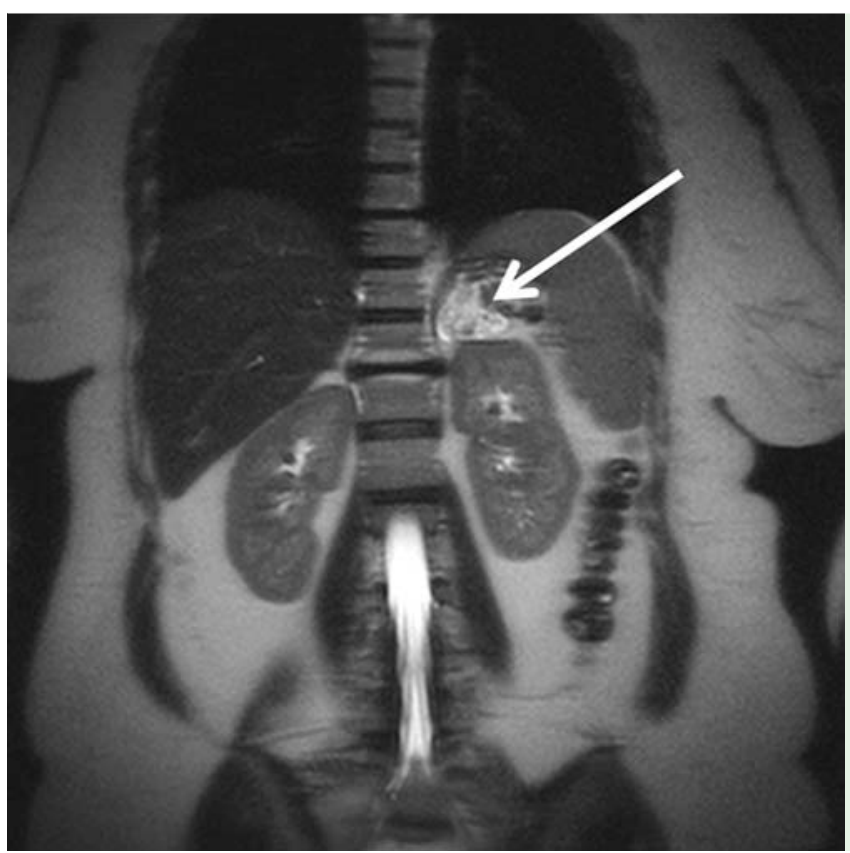

Fig. 1 Accidental finding of a suprarenal mass (arrow) on a magnetic resonance scan of the spine of a 46 -year-old obese woman with acute exacerbation of abdominal pain, recurring nausea and vomiting, and a history of chronic pain in the lumbar spine.

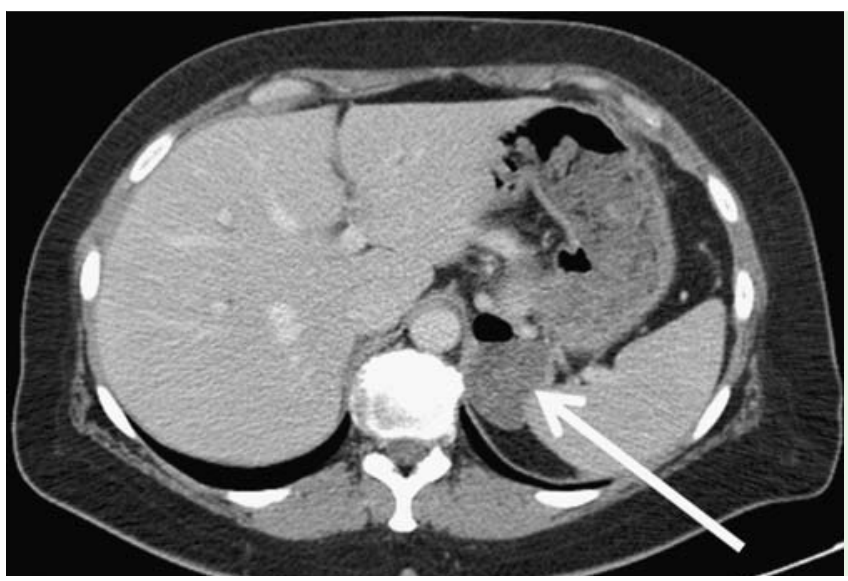

Fig. 2 Computed tomography scan showing gastric diverticulum (arrow).

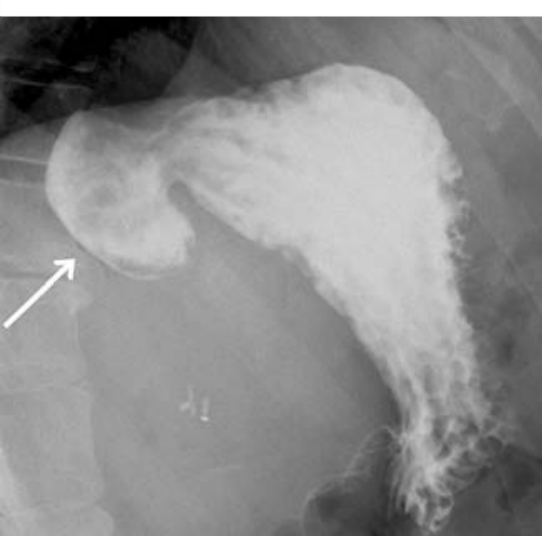

Fig. 3 Preoperative oral contrast study showing gastric diverticulum (arrow).
A 46-year-old obese female patient presented in the emergency room with acute exacerbation of abdominal pain, recurring nausea and vomiting, and a history of chronic pain in the lumbar spine. An external report of recently taken magnetic resonance images described a suspicious left adrenal mass ( $\bullet$ Fig. 1 ). The patient also had reflux symptoms and fetor ex ore. A history of rapid weight loss of $16 \mathrm{~kg}$ over the past 3 months and increasing fatigue raised suspicion for malignant disease. Assessment for pheochromocytoma was negative. A computed tomography (CT) scan excluded any malignancy and showed subcardial pouching of the dorsal gastric wall ( $\bullet$ Fig.2). Oral contrast swallow ( Fig.3) and endoscopy confirmed the diagnosis of a gastric diverticulum with an aperture of $35 \mathrm{~mm}$, filled with food remnants. Due to the clinical relevance of this benign finding, the patient was referred to general surgery. Using an endoscopic rendezvous technique, laparoscopic resection was carried out with an EchelonFlex60 Endopath Stapler (Ethicon Endo-Surgery, Cincinnati, Ohio, USA) under visual control from both sides of the diverticulum ( Fig.4). Histologic examination showed a full-thickness stomach specimen with slight chronic inflammation of otherwise normal gastric mucosa, indicative of a congenital diverticulum. Both the fetor and reflux symptoms ceased immediately after the surgery. A second contrast swallow on postoperative day 5 showed a patent suture and the patient was discharged thereafter.

Gastric diverticula are a rare entity of diverticula of the gastrointestinal tract. Due to their rarity, they are often mistaken for pathologies such as, most commonly, left adrenal masses [1,2]. Usually asymptomatic, they sometimes present with diffuse abdominal symptoms and gastric bleeding $[3,4]$. The preferred treatment is laparoscopic resection of the diverticulum, however, open approaches have also been reported [5]. A rendezvous technique as carried out in our department has not yet been described, but constitutes a safe treatment method.

Endoscopy_UCTN_Code_TTT_1AT_2AD

Competing interests: None

\section{B. Globke ${ }^{1}$, P. Fikatas ${ }^{1}$, A. Beck ${ }^{2}$, A. Adler ${ }^{3}$, S. C. Schmidt ${ }^{1}$}

${ }^{1}$ Department of General, Visceral and Transplantation Surgery, Campus Virchow-Klinikum, Charité - Universitätsmedizin Berlin, Berlin, Germany

2 Department of Radiology, Campus

Virchow-Klinikum, Charité - Universitätsmedizin Berlin, Berlin, Germany

${ }^{3}$ Department of Interdisciplinary Endoscopy, Division of Gastroenterology and Hepatology, Campus VirchowKlinikum, Charité - Universitätsmedizin Berlin, Berlin, Germany 

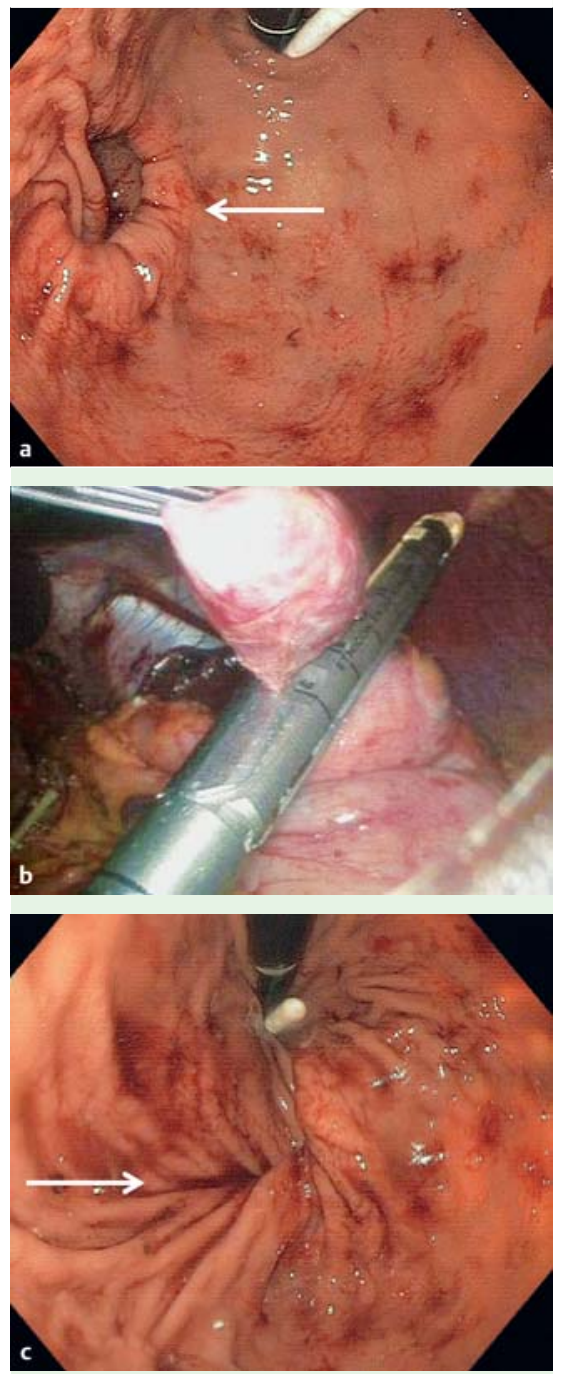

Fig. 4 a Intraoperative endoscopic visualization of the diverticulum aperture (arrow). b Laparoscopic view of diverticulum locked in GIA stapler. c Intraoperative endoscopic visualization after removal of the diverticulum (arrow).

\section{References}

1 Noguera JJ, Benito A, Hernandez-Sastre C et al. Gastric diverticulum mimicking cystic lesion in left adrenal gland. Urology 2009; 73 (Suppl. 05): 997-998

2 Kodera $R$, Otsuka F, Inagaki $K$ et al. Gastric diverticulum simulating left adrenal incidentaloma in a hypertensive patient. Endocr J 2007; 54 (Suppl. 06): 969-974

3 Perbet S, Constantin JM, Poincloux $L$ et al. Gastric diverticulum: a rare cause of hemorrhagic shock. Intensive Care Med 2008; 34 (Suppl. 07): 1353-1354

4 Lajoie A, Strum WB. Gastric diverticulum presenting as acute hemorrhage. Gastrointest Endosc 2008; 67 (Suppl. 01): 175 - 176

5 Elliott S, Sandler AD, Meehan JJ et al. Surgical treatment of a gastric diverticulum in an adolescent. J Pediatr Surg 2006; 41 (Suppl. 08): $1467-1469$

\section{Bibliography}

DOI http://dx.doi.org/

10.1055/s-0032-1326455

Endoscopy 2013; 45: E138-E139

(C) Georg Thieme Verlag KG

Stuttgart · New York

ISSN 0013-726X

\section{Corresponding author}

\section{B. Globke}

Charité Universitätsmedizin Berlin Klinik für Allgemein-, Viszeralund Transplantationschirurgie Campus Virchow-Klinikum Augustenburger Platz 1

13353 Berlin

Germany

Brigitta.Globke@charite.de 